\title{
Dirichlet and Neumann Problems for String Equation, Poncelet Problem and Pell-Abel Equation
}

\author{
Vladimir P. BURSKII ${ }^{\dagger}$ and Alexei S. ZHEDANOV ${ }^{\ddagger}$ \\ $\dagger$ Institute of Applied Mathematics and Mechanics NASU, Donetsk, 83114 Ukraine \\ E-mail:v30@dn.farlep.net \\ $\ddagger$ Donetsk Institute for Physics and Technology NASU, Donetsk, 83114 Ukraine \\ E-mail: zhedanov@yahoo.com
}

Received November 23, 2005, in final form March 20, 2006; Published online April 12, 2006

Original article is available at http://www.emis.de/journals/SIGMA/2006/Paper041/

\begin{abstract}
We consider conditions for uniqueness of the solution of the Dirichlet or the Neumann problem for 2-dimensional wave equation inside of bi-quadratic algebraic curve. We show that the solution is non-trivial if and only if corresponding Poncelet problem for two conics associated with the curve has periodic trajectory and if and only if corresponding Pell-Abel equation has a solution.
\end{abstract}

Key words: Dirichlet problem; Neumann problem; string equation; Poncelet problem; PellAbel equation

2000 Mathematics Subject Classification: 35L20; 14H70; 13B25

We will examine the Dirichlet problem for the string equation

$$
\begin{array}{ll}
\Phi_{x y}=0 & \text { in } \quad \Omega, \\
\left.\Phi\right|_{C}=0 & \text { on } \quad C=\partial \Omega,
\end{array}
$$

in a bounded semialgebraic domain, the boundary of which is given by some bi-quadratic algebraic curve given in the Euler-Baxter form $[1,13]$ :

$$
F(x, y)=x^{2} y^{2}+1+a\left(x^{2}+y^{2}\right)+2 b x y=0
$$

with real $a$ and $b$ that are subordinated to conditions of boundedness $a>0$ and nonvanishing $(|b|-a)^{2}>1$.

The Euler-Baxter curve (3) is a special case of generic bi-quadratic curve

$$
\sum_{i, k=0}^{2} a_{i k} x^{i} y^{k}=0
$$

with arbitrary 9 coefficients $a_{i k}$. In the general situation this curve has a genus $g=1$ and hence is birationally equivalent to an elliptic curve [7].

As far as we know, the John algorithm for such curve was first considered in [5], where it was shown that the corresponding discrete dynamical system is completely integrable and some solutions in terms of elliptic functions were presented in special cases.

In this paper we will concentrate mostly on analysis of the Euler-Baxter curve (3), because the generic curve (4) can be transformed to the form (3) by elementary linear rational transformations [8]

$$
x \rightarrow \frac{\alpha_{1} x+\beta_{1}}{\gamma_{1} x+\delta_{1}}, \quad y \rightarrow \frac{\alpha_{2} y+\beta_{2}}{\gamma_{2} y+\delta_{2}}
$$

with some coefficients $\alpha_{i}, \ldots, \delta_{i}, i=1,2$. 
Recall that the John algorithm is defined as follows [9].

Let $\Omega$ be an arbitrary bounded domain, which is convex with respect to characteristic directions, i.e. it has the boundary $C$ intersected in at most two points by each line that is parallel to $x$ - or $y$-axes. We start from an arbitrary point $M_{1}$ on $C$ and consider a vertical line passing through $M_{1}$. Obviously, there are exactly two points of intersection with the curve $C: M_{1}$ and some $M_{2}$. We denote $I_{1}$ an involution which transform $M_{1}$ into $M_{2}$. Then, starting from $M_{2}$, we consider a horizontal line passing through $M_{2}$. Let $M_{3}$ be the second point of intersection with the curve $C$. Let $I_{2}$ be corresponding involution: $I_{2} M_{2}=M_{3}$. We then repeat this process, applying step-by-step involutions $I_{1}$ and $I_{2}$. Denote $T=I_{2} I_{1}, T^{-1}=I_{1} I_{2}$. This transformation $T: C \rightarrow C$ produces a discrete dynamical system on $C$, i.e. an action of group $\mathbb{Z}$ and each point $M \in C$ generates an orbit $\left\{T^{n} M \mid n \in \mathbb{Z}\right\}$. This orbit can be either finite or infinite. The point $M$ corresponding to a finite orbit is called a periodic point and minimal $n$, for which $T^{n} M=M$, is called a period of the point $M$. F. John have proved several useful assertions, among which we point out the following one.

Sufficient condition of uniqueness. The homogeneous Dirichlet problem has no solutions apart form the trivial one in the space $C^{2}(\bar{\Omega})$ if the set of periodic points of $T$ on $C$ is finite or denumerable, in particular, if there are no periodic points on $C$.

Recall now the Poncelet algorithm [2]. Let $A$ and $B$ be two arbitrary conics (say, two ellipses, this cases is general and can be achieved by an appropriate projective transformation). We start from an arbitrary point $M_{1}$ on the conic $\mathrm{B}$ and pass a tangent to the conic $A$. This tangent intersects the conic $\mathrm{B}$ in another point $M_{2}$. Then we pass another tangent from the point $M_{2}$ to the conic $A$. We obtain then the point $M_{3}$ on the conic $B$. This process can be repeated generating a set of the points $M_{1}, M_{2}, \ldots, M_{n}, \ldots$ on the conic $B$. We denote also $L_{1}, L_{2}, \ldots$ corresponding tangential points on the conic $A$. The famous Poncelet theorem [2] states that if this algorithm is periodic for $M_{1}$ (i.e. $M_{1}=M_{N}$ for some $N=3,4, \ldots$ ) then this property does not depend on the choice of initial point $M_{1}$ on the conic $B$. Obviously, the same property is valid for the tangent points: $L_{1}=L_{N}$. Thus the periodicity property for the Poncelet problem depends only on a choice of two conics $A$ and $B$ and does not depend on a choice of the initial point.

Our crucial observation is the following. It appears that the John algorithm for the biquadratic curve $C$ is equivalent to the Poncelet algorithm for some two conics in a two-dimensional affine space.

Consider a transformation $V:(x, y) \rightarrow(u, v)$ given by the formulae $u=x+y ; v=x y$. This transformation converts the bisectrix $x=y$ into the parabola $D=\left\{(u, v) \mid v=u^{2} / 4\right\}$, moreover the left halfplane $x<y$ is transformed onto the exterior $D^{-}$of the parabola $D$ and the mapping is a diffeomorphism, the right halfplane $x>y$ is transformed onto the same exterior $D^{-}$of the parabola $D$, the points $(x, y)$ and $(y, x)$ have the same image. The set of vertical lines $x=x_{0}$ is transformed into the set $E$ of lines $v=x_{0} u-x_{0}^{2}$, having the parabola $D$ as their envelope. The set of horizontal lines $y=y_{0}$ is transformed also into the same set $E$ of lines (the lines $x=c$ and $y=c$ have the same image). The curve (3) is transformed into the quadric $B$ :

$$
v^{2}+a u^{2}+2(b-a) v+1=0 \quad \text { or } \quad(v+b-a)^{2}+a u^{2}=(b-a)^{2}-1 .
$$

Now, the John algorithm in the plane $x, y$ becomes the Poncelet algorithm in the plane $u, v$, because a movement along the line $x=c$ is converted to a movement along the tangent line to the parabola $D$ from a fixed point on the quadric $B$. The movement from the same point of the bi-quadratic curve $C$ along the line $y=c$ is converted to a movement along the second tangent line to the parabola $D$ starting form the same point of the quadric $B$. (Recall that from a generic point of a quadric $B$ there are exactly two tangent lines to another quadric $D$.) The converse statement is also valid. 
Note that in our previous paper [4] we proposed a slightly different way to establish a connection between the Poncelet problem and the boundary value problem (1), (2).

This result allows one to establish some important properties of the John algorithm for our curve $F(u, v)=0$. For example, the Poncelet theorem says then that the periodicity property of the John algorithm depends only on equation of the bi-quadratic curve (3) and does not depend on the choice of initial point on this curve.

This means that only one of two possibilities occurs:

(i) either all points of the curve $C$ are non-periodic. It follows from the Poncelet problem that in this case the set $T^{n} M$ is dense on $C$, it is said to be the transitive case;

(ii) or all points of the bi-quadratic curve (3) have the same finite period $N$.

Moreover, we can establish an explicit criterion for periodicity condition for the John algorithm (see below) and to demonstrate a relation with the famous algebraic Pell-Abel equation. This relation comes from the well-known Cayley criterion for the Poncelet problem. Recall that the Cayley criterion can be formulated as follows [2]. Let $f(\lambda)=\operatorname{det}(A-\lambda B)$ be a characteristic determinant for the one-parameter pencil of conics $A$ and $B$ presented in the projective form. In more details, assume that the conic $A$ has an affine equation $\phi_{A}(x, y)=0$. We then pass to the projective co-ordinates $x=\xi_{1} / \xi_{0}, y=\xi_{2} / \xi_{0}$ and present the equation of the conic $A$ in the form

$$
\sum_{i, k=0}^{2} A_{i k} \xi_{i} \xi_{k}=0
$$

with some $3 \times 3$ matrix $A$. Similarly, the projective equation for the conic $B$ has the form

$$
\sum_{i, k=0}^{2} B_{i k} \xi_{i} \xi_{k}=0
$$

with some $3 \times 3$ matrix $B$. We then define the polynomial $f(\lambda)=\operatorname{det}(A-\lambda B)$ of the third degree. Note that $f(\lambda)$ is a characteristic polynomial for the generalized eigenvalue problem for two matrices $A, B$. Calculate the Taylor expansion

$$
\sqrt{f(\lambda)}=c_{0}+c_{1} \lambda+\cdots+c_{n} \lambda^{n}+\cdots
$$

and compute the Hankel-type determinants from these Taylor coefficients:

$$
H_{p}^{(1)}=\left|\begin{array}{cccc}
c_{3} & c_{4} & \ldots & c_{p+1} \\
c_{4} & c_{5} & \ldots & c_{p+2} \\
\ldots & \ldots & \ldots & \ldots \\
c_{p+1} & c_{p+2} & \ldots & c_{2 p-1}
\end{array}\right|, \quad p=2,3,4, \ldots
$$

and

$$
H_{p}^{(2)}=\left|\begin{array}{cccc}
c_{2} & c_{3} & \ldots & c_{p+1} \\
c_{3} & c_{4} & \ldots & c_{p+2} \\
\ldots & \ldots & \ldots & \ldots \\
c_{p+1} & c_{p+2} & \ldots & c_{2 p}
\end{array}\right|, \quad p=1,2,3, \ldots
$$

Then the Cayley criterion [2, 7] is: the trajectory of the Poncelet problem is periodic with the period $N$ if and only if $H_{p}^{(1)}=0$ for $N=2 p$, and $H_{p}^{(2)}=0$ for $N=2 p+1$. For modern proof of the Cayley criterion see, e.g. [7]. Moreover, we have the following observation: the Cayley condition (6) coincides with a solvability criterion of the Pell-Abel equation

$$
A^{2}(\lambda)+\tilde{f}(\lambda) B^{2}(\lambda)=1
$$


with $\operatorname{deg} \tilde{f}=4, f(0)=0$ (for details concerning solvability of the Pell-Abel equation and its relations with other problems of mathematics see, e.g. papers by V.A. Malyshev [10, 11]) if one takes $f(x)=x^{4} \tilde{f}\left(x^{-1}\right)$. Recall that in the equation (8) the function $f$ is known and one should find unknown polynomials $A$ and $B$. The main problem here is to find conditions on the coefficient $f$ under which the equation has a solution $A, B$. We have the following proposition (a detailed analysis of this proposition will be published elsewhere):

The Poncelet problem is periodic with an even period iff corresponding the Pell-Abel equation is solvable.

Note that the case of odd period $2 N+1$ for the Poncelet problem corresponds to the case of the same period $2 N+1$ for the John algorithm, whereas the case of even period $2 N$ for the Poncelet problem corresponds to the case of period $N$ for the John algorithm.

Thus, in order to decide whether or not the John algorithm for the given bi-quadratic curve (3) is periodic we first pass from the John algorithm to the corresponding Poncelet problem and then apply the Cayley criterion.

Moreover, we have given the explicit solution of the John algorithm for the bi-quadratic curve (4) (see [4]):

$$
x_{n}=\phi\left(q\left(n-n_{1}\right)\right), \quad y_{n}=\psi\left(q\left(n-n_{2}\right)\right),
$$

where $\phi(z), \psi(z)$ are two different elliptic functions of the second order with the same periods; $n_{1}, n_{2}$ are some parameters depending on initial conditions. The parameter $q$ and the periods $2 \omega_{1}, 2 \omega_{2}$ of the elliptic functions $\phi(z), \psi(z)$ do not depend on initial conditions.

These considerations allow one to write down the general solution of the John algorithm in the form

$$
x_{n}=\kappa_{1} \frac{a_{1} \wp\left(q\left(n-n_{1}\right)+b_{1}\right.}{c_{1} \wp\left(q\left(n-n_{1}\right)+d_{1}\right.}, \quad y_{n}=\kappa_{2} \frac{a_{2} \wp\left(q\left(n-n_{2}\right)+b_{2}\right.}{c_{2} \wp\left(q\left(n-n_{2}\right)+d_{2}\right.}
$$

with some parameters $\kappa_{i}, a_{i}, b_{i}, c_{i}, d_{i}, n_{i}, i=1,2[4]$.

Periodicity condition for the John algorithm is

$$
q N=2 \omega_{1} m_{1}+2 \omega_{2} m_{2}
$$

where $N, m_{1}, m_{2}$ are integers.

For the special case of the Euler-Baxter biquadratic curve (3) we have a much simpler solution

$$
x_{n}=\sqrt{k} \operatorname{sn}\left(q\left(n-n_{0}\right), k\right), \quad y_{n}=\sqrt{k} \operatorname{sn}\left(q\left(n-n_{0}+1 / 2\right), k\right),
$$

where $n_{0}$ is an arbitrary parameter and parameters $q, k$ are easily determined from the parameters $a, b$ of the Euler-Baxter curve $[1,8]$.

Finally, we obtained new results concerning solutions for the boundary value problems for the string equation in case of periodicity of the John algorithm. For the Dirichlet problem we have the following result: Let $C$ be a nondegenerate bi-quadratic curve (3). The homogeneous Dirichlet problem (1), (2) has a nontrivial solution $\Phi \in C^{2}(\Omega)$ iff the John algorithm is periodic, i.e. the condition (10) is fulfilled. In this case there is an infinite set of linearly independent smooth solutions.

Besides, note the Dirichlet problem (2) has some nontrivial solution $u$ in Sobolev spaces iff the Neumann problem $\left.u_{\nu_{*}}^{\prime}\right|_{C}=0$ with the conormal $\nu_{*}$ for the equation (1) has a nonconstant solution $u$ [3].

Thus, the Poncelet problem and Pell-Abel equation are closely connected to the Neumann problem for the string equation as well. And the condition (10) is a criterion of existence of nontrivial solution for each of these problems. 
[1] Baxter R., Exactly solvable models in statistical mechanics, London, Academic Press, 1982.

[2] Berger M., Géométrie, CEDIC, Paris, 1978.

[3] Burskii V.P., Investigation methods of boundary value problems for general differential equations, Kyiv, Naukova Dumka, 2002 (in Russian).

[4] Burskii V.P., Zhedanov A.S., The Dirichlet and the Poncelet problems, RIAM Symposium No.16ME-S1 "Physics and Mathematical Structures of Nonlinear Waves" (November 15-17, 2004, Kyushu University, Kasuga, Fukuoka, Japan), 2004, 22-26, see here.

[5] Francoise J.P., Ragnisco O., An iterative process on quartics and integrable symplectic maps, in Symmetries and Integrability of Difference equations (1996, Canterbury), Editors P.A. Clarkson and F.W. Nijhoff, London Math. Soc. Lecture Note Ser., Vol. 255, Cambridge, Cambridge Univ. Press, 1999, 56-63.

[6] Granovskii Ya.I., Zhedanov A.S., Integrability of the classical XY chain, Pis'ma Zh. Eksper. Teoret. Fiz., 1986, V.44, 237-239 (English transl.: JETP Lett., 1986, V.44, N 5, 304-307).

[7] Griffiths P., Harris J., On a Cayley's explicit solution to Poncelet's porism, Enseign. Math. 2, 1978, V.24, $31-40$.

[8] Iatrou A., Roberts J.A.G., Integrable mappings of the plane preserving biquadratic invariant curves II, Nonlinearity, 2002, V.15, 459-489.

[9] John F., The Dirichlet problem for a hyperbolic equation, Amer. J. Math., 1941, V.63, 141-154.

[10] Malyshev V.A., Abel equation, Algebra i Analiz, 2001, V.13, N 6, 1-55 (English transl.: St. Petersburg Math. J., 2001, V.13, N 6, 893-938).

[11] Malyshev V.A., Periods of quadratic irrationalities, and torsion of elliptic curves, Algebra i Analiz, 2003, V.15, N 4, 177-203 (English transl.: St. Petersburg Math. J., 2004, V.15, N 4, 587-602).

[12] Sodin M.L., Yuditskii P.M., Functions least deviating from zero on closed subsets of the real axis, Algebra $i$ Analiz, 1992, V.4, N 2, 1-61 (English transl.: St. Petersburg Math. J., 1993, V.4, N 2, 201-249).

[13] Veselov A.P., Integrable systems with discrete time and difference operators, Funktsional. Anal. i Prilozhen., 1988, V.22, 1-13 (English transl.: Funct. Anal. Appl., 1988, V.22, N 2, 83-93).

[14] Whittacker E.T., Watson G.N., A course of modern analysis, Cambridge, 1927. 